\title{
Hydrogenic retention in irradiated tungsten exposed to high-flux plasma
}

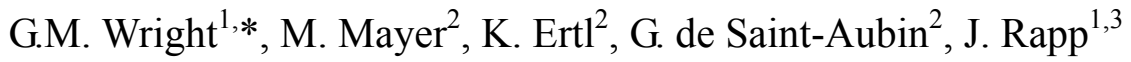

${ }^{1}$ FOM-Institute for Plasma Physics Rijnhuizen, EURATOM-FOM, A Member of the Trilateral Euregio Cluster, Postbus 1207, 3430 BE, Nieuwegein, The Netherlands.

${ }^{2}$ Max-Planck-Institut für Plasmaphysik, Association EURATOM, Postfach 1317, D-85741, Garching, Germany

${ }^{3}$ Insitute for Energy Research,FZ-Juelich,Association EURATOM, Trilateral Euregio Cluster, D-52425, Juelich, Germany.

*Main author email: wright@psfc.mit.edu

Two sets of identical tungsten (W) targets are irradiated at $300 \mathrm{~K}$ with $12.3 \mathrm{MeV} \mathrm{W}^{4+}$ ions to peak damage levels ranging from $0.5-10$ displacements per atom (dpa). This results in a damage profile that is peaked at $\sim 0.8 \mu \mathrm{m}$ and extends to a depth of $\sim 1.5 \mu \mathrm{m}$. Both sets of targets are exposed to high-density $\left(\mathrm{n}_{\mathrm{e}, \mathrm{center}}=3 \times 10^{20} \mathrm{~m}^{-3}\right.$ ), lowtemperature $\left(\mathrm{T}_{\mathrm{e}, \text { center }}=1.6 \mathrm{eV}\right)$ deuterium (D) plasma in Pilot-PSI. One set of irradiated targets is exposed at high surface temperatures $\left(T_{\mathrm{W}}=950-680 \mathrm{~K}\right)$ and the other at low surface temperatures $\left(\mathrm{T}_{\mathrm{W}}=480 \mathrm{~K}-340 \mathrm{~K}\right)$. The surface temperature is determined by the local plasma conditions. Nuclear reaction analysis (NRA) is used to determine the $\mathrm{D}$ depth profiles at specific radial locations, thus giving a surface temperature scan of the D retention in the damaged W. Global retention is determined by thermal desorption spectroscopy, which yields total $\mathrm{D}$ retained in the target and also gives information of the different types of lattice defects that are trapping the $\mathrm{D}$ in the $\mathrm{W}$ lattice. The main results are that there is no measurable difference between the different dpa levels, implying a saturation of the retention enhancement at a level $\leq 0.5 \mathrm{dpa}$. For both irradiated and unirradiated tungsten, a peak in the retention is seen at $\mathrm{T}_{\mathrm{W}}=480 \mathrm{~K}$, however the $\mathrm{W}^{4+}$ irradiation clearly enhances the retention. This enhancement is also temperature dependent and increases with increasing surface temperature up to an enhancement by a factor of $15-23$ at $T_{W}=950 \mathrm{~K}$. At the lowest surface temperatures, a fluence dependence appears since the implanted deuterium is diffusion limited to only a small fraction of the irradiated zone. TDS spectra show an enhancement of both low energy trap sites and high energy trap sites. For these conditions, diffusion-limited, low fill fraction trapping determines the hydrogenic retention of the W.

PACS: 52.40.Hf, 28.52.Fa, 28.52.Cx, 78.40.Kc, 61.80.-x 


\section{Introduction}

In future burning fusion devices, the presence of $14.1 \mathrm{MeV}$ neutrons from the deuterium-tritium (D-T) fusion reaction can significantly change many of the physical properties of the plasma-facing materials. The neutron irradiation of the materials can lead to embrittlement, $\mathrm{He}$ or $\mathrm{H}$ production, and degradation of thermoconductivity. Neutron irradiation will also lead to the formation of defects and voids in the lattice as the neutrons collide with the lattice atoms as they pass through the material.

Due to the difficult and time-consuming nature of neutron irradiation experiments and the difficulties in handling activated neutron-irradiated targets, high-energy ions are often used as a proxy for neutron damage. Several studies have shown that irradiation of tungsten with high energy (MeV-range) ions can enhance retention significantly [1-6]. There are still a large number of uncertainties and inconsistencies in the understanding of the influence of this irradiation damage on hydrogenic retention. It is well documented that the irradiation damage results in additional lattice defect sites that trap hydrogen thus increasing the hydrogenic retention of the material. The damage profile induced in the lattice by the high energy ions is limited in depth by the implantation range of the irradiating ion (typically on the order of microns) as opposed to neutron irradiation where the damage is uniformly distributed across the entire depth of a typical target thickness. The irradiating ion damage also has a non-uniform damage distribution with in the implantation zone, with a peak in damage occurring near the end of range for the irradiating ions. The depth distribution of the damage profiles can be

simulated by the TRIM ${ }^{1}$ code to a high degree of accuracy, although the absolute value of the damage is a matter of debate.

The influence of surface temperature and plasma exposure conditions on the enhancement of retention due to irradiation damage is currently unknown or unclear. The goal of this investigation is to examine the enhancement of retention in irradiated tungsten over a range of damage levels and surface temperatures when exposed to high-density, low-temperature plasmas that are expected in the divertor of future fusion devices.

\section{Experiment}

\subsection{Materials}


The $\mathrm{W}$ used for this investigation was polycrystalline $\mathrm{W}$ (purity of $99.97 \%$ ) purchased from Midwest Tungsten. The targets were disks of a $20 \mathrm{~mm}$ diameter and $1 \mathrm{~mm}$ thickness. There was no pre-annealing or polishing of the tungsten targets before they were exposed in either Pilot-PSI or irradiated at IPP-Garching. The "as received" conditions were used to best re-create a typical pure tungsten surface found in a tokamak divertor.

\section{$2.212 .3 \mathrm{MeV} \mathrm{W}^{4+}$ Irradiation}

W targets were irradiated with $12.3 \mathrm{MeV} \mathrm{W}^{4+}$ ions at the tandem ion accelerator at IPP-Garching. The target was water-cooled during irradiation to ensure that the irradiation temperature remained at $300 \mathrm{~K}$. The irradiated area was circular with a diameter of $12 \mathrm{~mm}$. The irradiating beam spot was focussed to $\sim 1 \mathrm{~mm}$ diameter spot size and then raster scanned across the irradiated zone so uniform exposure was obtained across the surface.

TRIM simulations are used to predict the damage rate and damage profile from the irradiation (see figure 1). For these simulations a displacement energy of $40 \mathrm{eV}$ was used rather than the default of $25 \mathrm{eV}$ for TRIM, which is much too low for the W lattice. Other displacement energies can be found in literature (such as the suggested $E_{d}=68 \mathrm{eV}$ in [7]), but an uncertainty in $E_{d}$ affects only the total level of predicted displacements per atom (dpa) and not the distribution of this damage in the TRIM simulation. TRIM simulations indicate a peak in the damage at $\sim 0.8 \mu \mathrm{m}$ and no damage at a depth beyond $\sim 1.5 \mu \mathrm{m}$. Targets were irradiated to peak damage levels of $0.5,1.0,2.0$, and $10 \mathrm{dpa}$. In addition one target from each set was irradiated to $1 \mathrm{dpa}$ on both the front and backside of the target to check for permeation of deuterium during plasma exposure.

\subsection{Deuterium plasma exposure in Pilot-PSI}

Irradiated targets were then taken to FOM-Rijnhuizen where a $16 \mathrm{~mm}$ diameter circular area is exposed to deuterium (D) plasma in Pilot-PSI [8]. The plasma electron density $\left(\mathrm{n}_{\mathrm{e}}\right)$ and temperatures $\left(\mathrm{T}_{\mathrm{e}}\right)$ are measured with Thomson scattering. In Pilot-PSI, $\mathrm{n}_{\mathrm{e}}$ and $\mathrm{T}_{\mathrm{e}}$ follow a peaked distribution across the plasma column width leading to a peaked distribution in plasma flux density. Exposure conditions were kept constant with a central $n_{e}$

of $(3.0 \pm 0.2) \times 10^{20} \mathrm{~m}^{-3}$ and a central $\mathrm{T}_{\mathrm{e}}$ of $1.6 \pm 0.2 \mathrm{eV}$. The full distribution of $\mathrm{n}_{\mathrm{e}}$ and $\mathrm{T}_{\mathrm{e}}$ can be seen in figure 2 . Total exposure time was $80 \mathrm{~s}(4 \times 20 \mathrm{~s}$ pulse $)$ however one sample was exposed for $160 \mathrm{~s}(8 \mathrm{x} 20 \mathrm{~s}$ pulse $)$ and

\footnotetext{
${ }^{1}$ WwW.srim.org
} 
one for $240 \mathrm{~s}$ ( $12 \times 20 \mathrm{~s}$ pulse) to investigate fluence dependence. There is $\sim 10$ minute break between each pulse to allow for magnet cooling, but the target is quenched and held at room temperature during this time.

Heat fluxes, and thus surface temperatures, are determined entirely by the plasma and thus also follow a peaked profile. The central temperature $(\sim 1 \mathrm{~mm}$ diameter measurement spot) is measured to a high degree of accuracy and reliability with a multi-wavelength spectropyrometer. The temperature distribution is then obtained with a 2-D IR camera using the central temperature as a calibration point. In this investigation two temperature ranges were investigated by the use of different target holders. The high temperature scenario $\left(\mathrm{T}_{\mathrm{W}}>650 \mathrm{~K}\right)$ is obtained by mechanically clamping the target to a water-cooled copper heatsink using a layer of HITHERM grafoil to improve thermal contact. For the high temperature scenario (HTS), central temperatures of $950 \pm 30 \mathrm{~K}$ were measured with temperatures decreasing to $650 \pm 30 \mathrm{~K}$ at the edge of the exposed area. The low temperature scenario $\left(\mathrm{T}_{\mathrm{W}}<500 \mathrm{~K}\right)$ is obtained by having the water cooling in direct contact with the backside of the target, thus all issues with thermal contact or poor thermal conductivity are avoided. For the low temperature scenario (LTS), central temperatures of $480 \pm 20 \mathrm{~K}$ were measured with temperatures decreasing to $340 \pm 35 \mathrm{~K}$ at the edge of the exposed area. For the LTS only the 2-D IR camera could be used since it was below the detectable limit for the spectropyrometer. At the very cold edges of the target, the IR camera yields artificially high temperatures since the signal is dominated by IR reflections. To correct for this, it was assumed since the plasma exposure conditions were identical in both cases, the temperatures at the cold edge were extrapolated from the same relative shape of the temperature profile as was measured for the HTS.

Since the power to the target is brought entirely by the plasma, this makes the thermal evolution of targets in Pilot-PSI somewhat unique when compared to similar linear plasma devices. Due to the strong heating from the plasma and strong cooling from the target holder, thermal equilibrium in the target is reached in $\sim 1 \mathrm{~s}$ once the plasma exposure is initiated. When the plasma is removed, the target is quenched back to near room temperature in 1-2 s. This effectively "freezes" the implanted deuterium in place, until diffusion can resume in the following shot when the temperature is elevated again. This rapid quenching after the plasma exposure is what makes the sequential plasma pulses essentially equivalent to one continuous shot for the full exposure time.

The full temperature profile for HTS and LTS can be seen in figure 3 and a summary of exposure conditions at various radial locations are listed in table 1 . The plasma column can be assumed to be poloidally 
uniform since it is rotating at a high frequency [9]. All targets are electrically floating during exposure so fluxes are calculated assuming the Bohm criterion.

\begin{tabular}{|c|c|c|c|c|c|c|}
\hline $\begin{array}{c}\text { Radial } \\
\text { Position }\end{array}$ & $\begin{array}{c}\mathrm{n}_{\mathrm{e}} \\
\left(\times 10^{20} \mathrm{~m}^{-3}\right)\end{array}$ & $\mathrm{T}_{\mathrm{e}}(\mathrm{eV})$ & $\begin{array}{c}\Gamma_{\text {Bohm }} \\
\left(\times 10^{24} \mathrm{~m}^{2} / \mathrm{s}\right)\end{array}$ & $\begin{array}{c}\mathrm{D} \text { fluence } \\
\left.10^{25} \mathrm{~m}^{2}\right)\end{array}$ & $\begin{array}{c}\mathrm{T}_{\text {surf }}(\mathrm{K}) \\
\mathrm{LTS}\end{array}$ & $\begin{array}{c}\mathrm{T}_{\text {surf }}(\mathrm{K}) \\
\mathrm{HTS}\end{array}$ \\
\hline $\mathbf{0 ~} \mathbf{~ m m}$ & $\mathbf{3 . 0} \pm \mathbf{0 . 3}$ & $\mathbf{1 . 6} \pm \mathbf{0 . 1}$ & $\mathbf{2 . 7} \pm \mathbf{0 . 3}$ & $\mathbf{2 2} \pm \mathbf{2}$ & $\mathbf{4 8 0} \pm \mathbf{2 0}$ & $\mathbf{9 5 0} \pm \mathbf{3 0}$ \\
\hline $\mathbf{3 ~} \mathbf{~ m m}$ & $\mathbf{2 . 4} \pm \mathbf{0 . 2}$ & $\mathbf{1 . 3} \pm \mathbf{0 . 1}$ & $\mathbf{1 . 9} \pm \mathbf{0 . 2}$ & $\mathbf{1 5} \pm \mathbf{2}$ & $\mathbf{4 1 0} \pm \mathbf{2 0}$ & $\mathbf{8 0 0} \pm \mathbf{3 0}$ \\
\hline $\mathbf{5 ~} \mathbf{~ m m}$ & $\mathbf{1 . 4} \pm \mathbf{0 . 2}$ & $\mathbf{1 . 2} \pm \mathbf{0 . 1}$ & $\mathbf{1 . 1} \pm \mathbf{0 . 2}$ & $\mathbf{9} \pm \mathbf{2}$ & $\mathbf{3 6 0} \pm \mathbf{2 5}$ & $\mathbf{7 0 0} \pm \mathbf{3 0}$ \\
\hline $8 \mathrm{~mm}$ & $0.5 \pm 0.1$ & $0.8 \pm 0.1$ & $0.3 \pm 0.1$ & $2.4 \pm 0.8$ & $340 \pm 25$ & $650 \pm 40$ \\
\hline
\end{tabular}

Table 1: Exposure conditions for $W$ targets in Pilot-PSI at various radial locations. The bolded values represent locations within the $W^{4+}$ irradiated zone. An exposure time of $80 \mathrm{~s}$ is assumed for the fluence. Uncertainties are indicative of the spread of values measured for all targets rather than accuracy of the measurement technique.

\section{$2.3 \mathrm{D}\left({ }^{3} \mathrm{He}, \mathrm{p}\right) \alpha$ Nuclear Reaction Analysis}

Nuclear reaction analysis (NRA) allows for the determination of local concentrations of D retention in the $\mathrm{W}$ targets. Thus by scanning the NRA analysis spot $(\sim 1 \mathrm{~mm}$ diameter $)$ across the target, retention can be plotted as a function of varying parameters. NRA was performed at IPP-Garching. Multiple ${ }^{3} \mathrm{He}$ ion energies were used to further refine the depth profiling [10]; energies of 1.0, 1.4, 1.8, and 2.5 $\mathrm{MeV}$ were used to give a depth profile with $\sim 0.5 \mu \mathrm{m}$ resolution to a depth of $4 \mu \mathrm{m}$. The $\mathrm{D}$ concentrations are then integrated over the depth to give the total retention in the first $4 \mu \mathrm{m}$ of the surface. Depth profiles were simulated and fit with the SIMNRA program [11]. The analysis points were scanned across the targets and data taken at radial positions of $0,3,5$, and $8 \mathrm{~mm}$.

\subsection{Thermal desorption spectroscopy}

After NRA, the targets returned to FOM-Rijnhuizen for thermal desorption spectroscopy (TDS). The W target is clamped to a ceramic heater and heated at a constant rate of $1 \mathrm{~K} / \mathrm{s}$ up to a temperature of $1273 \mathrm{~K}$. A quadrupole mass spectrometer (QMS) monitors the mass $4\left(\mathrm{D}_{2}\right)$ and mass 3 (HD) signal in the residual gas in the chamber to determine the amount of deuterium out-gassed during the temperature ramp. The absolute sensitivity of the QMS is measured using calibrated leaks of $\mathrm{D}_{2}$ and $\mathrm{H}_{2}$. The sensitivity to mass 3 is taken as the average of the sensitivity between mass $2\left(\mathrm{H}_{2}\right)$ and mass 4 .

TDS yields the total retention of the target from the entire bulk but gives no information on the spatial distribution of the $\mathrm{D}$ in the $\mathrm{W}$. However, analysis of the desorption spectra can reveal information of the trap 
types that are present in the W. Thus TDS is an ideal compliment to NRA since it yields trap type information and total global retention, while NRA reveals local and spatially resolved retention in the near surface.

\section{Results and discussion}

\subsection{Spatial and depth distribution of $D$}

By scanning the NRA analysis point across the targets, this yields a 1D scan of D retention across the surface and a depth distribution over the first $4 \mu \mathrm{m}$ of the surface at these locations. The integrated D concentrations over the first $4 \mu \mathrm{m}$ are plotted as a function of radial distance on the target for both the high temperature scenario (HTS) and low temperature scenario (LTS) in figure 4. The local surface temperature is overlaid on the same plot and the relationship between surface temperature and retention becomes immediately clear. The retention is clearly decreasing with increasing temperature for the HTS. The low retention values at the $8 \mathrm{~mm}$ position are likely due to partial shadowing of the target from the plasma since this is the location of the edge of the molybdenum clamping ring that is used to clamp the target to the heat sink. This trend of strongly decreasing retention at these temperatures is not unexpected [12-13].

At all temperatures in the HTS there is a clear enhancement of retention due to the irradiation damage, however there is no significant difference in the retention for the different dpa levels, with the exception of the $0.5 \mathrm{dpa}$ case. However, upon closer inspection, the $0.5 \mathrm{dpa} \mathrm{W}$ target exposure was actually exposed at a surface temperature $\sim 150 \mathrm{~K}$ higher than all other targets, likely due to poor thermal contact with the heat sink. This temperature discrepancy was recorded on both the spectropyrometer and the 2-D IR camera.

The indications are that the enhancement of retention due to the irradiation damage is saturated at peak damage levels $\geq 0.5 \mathrm{dpa}$, also in good agreement with [1]. This is confirmed by the data from the LTS (figure 5) where clearly all different dpa levels have identical retention levels. However with the LTS, the trend with temperature is reversed and retention now increases with increasing surface temperature. The retention at the centre of the target $\left(T_{W}=480 \mathrm{~K}\right)$ is a factor of $\sim 3$ higher than the retention closer to the edge $\left(T_{W}=360 \mathrm{~K}\right)$. It is still clear that retention has been enhanced due to the irradiation damage.

Plotting the $\mathrm{D}$ retained in the first $4 \mu \mathrm{m}$ of the surface as a function of local temperature reveals a maximum in retention at $\mathrm{T}_{\mathrm{W}} \sim 500 \mathrm{~K}$ (see figure 5), although the temperature region between $500-650 \mathrm{~K}$ lacks 
any data. This maximum appears in both irradiated and unirradiated targets and has also been seen in other work $[3,14,15]$, although the actual peak can range from 400-600 K. It must also be noted that other parameters such as plasma flux, and thus plasma fluence, are not held constant in the data shown in figure 5. Table 1 demonstrates the differences in plasma flux that correspond to the different local W surface temperatures. However, previous work has shown no dependence of retention on plasma flux or fluence for unirradiated targets in the high temperature regime $[16,17]$. The possibility of a flux or fluence dependence in the low temperature regime is addressed later in this report.

This peak in retention is often explained as the influence and competition between diffusion and thermal de-trapping (e.g. the rate at which D escapes trap sites as bulk temperature is increased). An examination of the depth profiles from NRA makes this relationship clearer. The D depth profiles for both irradiated and unirradiated targets at various local $\mathrm{W}$ temperatures are shown in figure 6. From these depth profiles it is clear that the low retention at high temperatures is due to increased thermal de-trapping at these temperatures as is evidenced by the low local concentrations even for the irradiated targets. Note how the local concentrations increase as temperature moves from $950 \mathrm{~K}$ to $480 \mathrm{~K}$. Since the irradiation conditions were the same for all targets we know the initial trap concentration will be the same for all targets in the irradiated zone (retention outside the irradiation zone is not a significant factor), however the higher temperature allows for the thermal detrapping rate to equate to the trapping rate at a lower concentration of trapped $\mathrm{D}$ as can be seen in equation 1 where both $\langle\sigma v\rangle_{\text {trap }}$ and $\langle\sigma\rangle_{\text {de-trap }}$ are functions of temperature.

$$
n_{\text {trap }, \text { empty }} \cdot n_{D, \text { solute }}\langle\sigma\rangle_{\text {trap }}=\left(n_{\text {trap }, \text { filled }}\right)^{2}\langle\sigma\rangle_{\text {de-trap }}
$$

At higher temperatures, trap annealing (e.g. the removal of trap sites from the material) may begin to play a role in this balance as well, since this will reduce the number of available empty trap sites, $\mathrm{n}_{\text {trap,empty. }}$ However, since trap annealing typically takes place on longer timescales, this is thought to be a second-order effect.

Clearly the implanted deuterium has diffused throughout the entire irradiated zone for $\mathrm{T}_{\mathrm{W}} \geq 480 \mathrm{~K}$ since an enhancement in retention is seen across the full $1.5 \mu \mathrm{m}$ irradiation depth. At the lowest temperature $\left(\mathrm{T}_{\mathrm{W}}=360\right.$ $\mathrm{K})$, thermal de-trapping will be low, so the local concentrations should be high but now diffusion is the limiting factor. We can see that beyond the first $0.5 \mu \mathrm{m}$ of the surface, the retention between the irradiated and 
unirradiated targets is approximately equivalent. This implies that the implanted D has not diffused through the irradiation zone to fill all available traps there. The modest local D concentration in the first $0.5 \mu \mathrm{m}$ of the surface $(\sim 1.0$ at. $\%)$ may also be due to the limited diffusion. If the typical diffusion length scale of the implanted deuterium during the $80 \mathrm{~s}$ exposure time is less than the depth resolution of NRA then the depth "slab" in the NRA depth profile may not be fully permeated by the implanted D. Since the local concentration is the integrated concentration across this slab, this may result in a lower average D concentration in a slab that is not fully permeated with D.

This can be confirmed by exposing irradiated targets to longer exposure times (e.g. higher plasma fluences) in Pilot-PSI. This was done for targets irradiated to 2 dpa and exposed in Pilot-PSI for 160 ( 8 x $20 \mathrm{~s})$ and $240 \mathrm{~s}(12 \times 20 \mathrm{~s})$ for the LTS. Figure 7 shows the resulting depth profiles at the center $\left(\mathrm{T}_{\mathrm{W}}=480 \mathrm{~K}\right)$ and 5 mm off center $\left(T_{W}=360 \mathrm{~K}\right)$. It can be seen that for $T_{W}=360 \mathrm{~K}$, the longer exposure times allow the $\mathrm{D}$ to diffuse deeper into the irradiated zone, although even after $240 \mathrm{~s}$ it is still not permeated fully. Also the local concentration in the first "slab" of the depth profile increases with longer exposure time and after $240 \mathrm{~s}$ the local concentration is equivalent to that seen in the $T_{W}=480 \mathrm{~K}$ case. Also of interest is that at $T_{W}=480 \mathrm{~K}$, an increase in exposure time/plasma fluence results in no increase in retention, implying that the irradiated zone is fully permeated and fully saturated at a local D concentration of $1.2-1.5$ at. $\%$.

So for these irradiated targets, it is clear that in situations where the surface temperature is $\geq 480 \mathrm{~K}$, the maximum retention is determined by the equilibrium between trapping and thermal de-trapping rates since the irradiated zone is fully permeated after the $80 \mathrm{~s}$ exposure time used in this investigation. For temperatures $\leq 360$ $\mathrm{K}$, the retention is clearly limited by the diffusion of implanted $\mathrm{D}$ into the irradiated zone. Until the irradiated zone is fully permeated, the maximum retention will not be reached. We can not predict the maximum local concentration at $\mathrm{T}_{\mathrm{W}}=360 \mathrm{~K}$ since the local concentration at the surface continues to increase with exposure time. Since the $\mathrm{D}$ retention at $\mathrm{T}_{\mathrm{W}}=410 \mathrm{~K}$ is lower than at $\mathrm{T}_{\mathrm{W}}=480 \mathrm{~K}$ for the $80 \mathrm{~s}$ exposure time (see figure 5), it can also be guessed that at $410 \mathrm{~K}$, the irradiated zone is still not fully permeated by the implanted $\mathrm{D}$, but we do not have depth profiles at this temperature to confirm this.

For the targets that were irradiated on both sides, no D was detected on the backside of the target for either the HTS or the LTS. This means the D did not fully permeate the $1 \mathrm{~mm}$ thick W targets during the $80 \mathrm{~s}$ 
total exposure time, or that all trap sites were annealed from the target before they were reached and "stabilized" by the implanted deuterium. Simple calculations using the diffusion rate of $\mathrm{H}$ in $\mathrm{W}[18]$ indicate that the characteristic diffusion length (i.e. $\mathrm{L}=[\mathrm{D} \cdot \mathrm{t}]^{1 / 2}$ ) is less than $1 \mathrm{~mm}$ for the HTS temperatures. So it is expected that there is no permeation of the target in $80 \mathrm{~s}$.

While the irradiation damage is the dominant source of trap production in these experiments, it should be mentioned that the high-flux plasma can also have an impact on the retention, as is evidenced by the unirradiated targets. Many previous studies have postulated that high rates of ion implantation into low solubility metals, such as $\mathrm{W}$, can result in the production of trap sites through the super-saturation of the implantation zone creating stresses in the lattice [15,19-21]. Previous work on Pilot-PSI has shown this is not a strong contributor in the high-temperature regime [16], likely due to high hydrogenic diffusion rates at these temperatures. At lower temperatures, NRA analysis shows that local concentrations of D in un-irradiated targets has increased when compared to irradiated targets (Fig. 6). This perhaps indicates that the plasma is playing a greater role in terms of trap production at lower exposure temperatures.

\subsection{Hydrogenic retention enhancement due to $12.3 \mathrm{MeV} \mathrm{W}^{4+}$ irradiation}

The area of most interest is quantifying the degree of retention enhancement experienced in the tungsten since this is an important factor in understanding fuel retention in burning plasma devices. The most accurate way to quantify the enhancement is by comparing the local D concentrations from the depth profiles. We take the depth "slab" at 0.5-1.0 $\mu \mathrm{m}$ for this comparison since the first "slab" in the surface has a higher concentration of natural/inherent traps (e.g. traps produced from fabrication or machining of the targets) and this may affect the contributions from the irradiation, also the second slab contains the peak in damage so it has the most constant damage level. By comparing an irradiated case (we use 2 dpa case here) to the unirradiated case, the enhancement factor $\left(D_{\text {retained }}\right.$ at $2 \mathrm{dpa} / \mathrm{D}_{\text {retained }}$ at $0 \mathrm{dpa}$ ) can be plotted as a function of temperature (see figure 8).

The interesting trend here is that the enhancement factor increases with increasing target temperature. In fact at $T_{\mathrm{W}}=950 \mathrm{~K}$, when all data from literature states the retention should be virtually zero [16,17], we measure the highest retention enhancement with the retention being enhanced by a factor of 15-23. We also measure local D concentrations of $0.3-0.5$ at \%, which are at least an order of magnitude higher than anything seen at this temperature previously $[16,17]$. What is also interesting is that other work has shown that plasma exposure at 
$773 \mathrm{~K}$ results in virtually no enhancement of retention [2]. It is explained that this is above the annealing temperature for vacancies [22] so the induced damage from the ion irradiation is annealed out. However this appears not to be the case in this scenario. One possible explanation is that the high flux plasma in Pilot-PSI allows the implanted D to permeate the damaged zone very quickly and keeps the traps filled since the concentration of solute $\mathrm{D}$ will be high (see equation 1). These filled traps could be more difficult to anneal therefore allowing them to survive at such high temperatures. Another potential explanation is that the retention at high exposure temperature is a result of the thermal evolution at the targets. Since the target is quickly quenched back to approximately room temperature in 1-2 s after the plasma shot ends, much of the implanted deuterium in solution will be deep in the material with a much lower diffusion rate due to the rapid drop in temperature. A solute $\mathrm{D}$ atom is much more likely to populate a nearby empty trap site than it is to diffuse to a surface, recombine and escape. This would mean that much of the trapping takes place after the plasma shot. This has also been used to explain the presence of a desorption peak in the TDS spectra at a temperature below the exposure temperature [17]. This high enhancement seen at such high temperatures is one of the key results of this investigation. While the exact cause is still uncertain, this could have serious implications for fuel retention in fusion reactors which are expected to have high neutron fluxes (high damage rates) and operate with "hot walls" (e.g. $\left.\mathrm{T}_{\text {wall }} \sim 900 \mathrm{~K}\right)$.

\subsection{Deuterium trapping in irradiated $W$}

The NRA data yields an understanding of the amount of $\mathrm{D}$ retained and the location of that trapped $\mathrm{D}$, but it gives no information at all about what type of traps are created and how the D is distributed over different trap types. TDS can yield this information. Figure 9 shows thermal desorption spectra from the irradiated (2 dpa) and unirradiated cases for the LTS and HTS. Distinct desorption peaks correspond to distinct trap energies (e.g. the energy required for the $\mathrm{D}$ to escape the trap and return to solution). Low temperature desorption peaks indicate the presence of low energy trap sites and high temperature peaks indicate the presence of high energy trap sites. Desorption from deep in the bulk can also lead to high temperature desorption peaks but since we know the irradiation damage only extends $1.5 \mu \mathrm{m}$ into the surface, the depth of the trapping is not likely to affect the desorption spectra. 
It is clear that the irradiation damage introduces a high temperature desorption peak centred at $\sim 850 \mathrm{~K}$ for both the LTS and HTS. It is also interesting to note that the low temperature desorption peak at $\sim 500 \mathrm{~K}$ that was present in the unirradiated targets was also enhanced by the irradiation damage, although this is only evident in the LTS data. There may also be an introduction of a third, smaller peak at $\sim 600 \mathrm{~K}$ but it is hard to determine due to the large size of the surrounding peaks.

The strongest influence of the irradiation is the introduction of the high temperature desorption peak. This corresponds to a high energy trap site. Literature states that desorption peaks near $900 \mathrm{~K}$ correspond to trap energies of $\sim 2.1 \mathrm{eV}$ [21]. These traps are typically voids [23] and the $\mathrm{D}$ is trapped at the inner surface of the internal void. These voids are often formed from vacancies clustering together to form small microvoids. The magnitude of the high temperature peak is a factor of $\sim 2$ greater for the HTS than the LTS. However, we have seen that for the LTS, the irradiated zone is not fully permeated at all locations, so there is likely a considerable population of trap sites that remained empty during the plasma exposure. This is confirmed when we examine the TDS spectra from the targets with longer exposure time as seen in figure 10. We see the high temperature desorption peak "filling up" as the D begins to fully permeate the irradiated zone. We also see the peak at 600 $\mathrm{K}$ becoming more prominent.

The trends in the low temperature desorption peak are also of interest. Desorption peaks between 500$600 \mathrm{~K}$ are attributed to trapping at single vacancies and have trap energies ranging from 1.1-1.4 eV [20]. We see no enhancement of this peak in the HTS between irradiated and unirradiated targets. However given the low trap energy corresponding to this peak this is not unexpected. Given that $\langle\sigma v\rangle_{d e-t r a p}$ is exponentially dependent on trap energy, there will be a very high de-trapping rate for these trap sites during the HTS exposures. Plus, literature has established that at these temperatures vacancies can also be annealed from the material [19]. So the highest probability is that these trap sites remain mostly empty or are annealed during the HTS plasma exposure. For the LTS exposures these traps are much more easily populated. Although from the TDS spectra it is clear that access to these low energy traps is also diffusion limited meaning they are also likely distributed fairly evenly through out the irradiated zone.

For the LTS there is a clear increase in overall retention as a function of plasma fluence/exposure time. The total $\mathrm{D}$ atoms retained for the spectra shown in figures 9 and 10 can be found in table 2 . If the increase of 
retention at the higher exposure times was merely diffusion limited then the retention would increase as $\mathrm{t}^{1 / 2}$, but the increase in retention is stronger than the expected square root dependence. This implies there is also a plasma fluence dependence as well as a time dependence. This may be also connected to the fact that we expose the target in $20 \mathrm{~s}$ pulses rather than one long continuous exposure. This is due to the limited cooling of the copper magnets on Pilot-PSI, but the impact of pulsed vs. continuous operation is a topic of future research. Also of interest is that the two 0 dpa cases have almost identical global retention (Table 1), despite different measured local D concentrations (Fig. 6). This can likely be attributed to diffusion-limited retention for the LTS, and deeper penetration but higher thermal de-trapping rates and less trap production via high flux plasma exposure for the HTS scenario.

The TDS data is consistent with the retention behaviour as determined from the NRA data. The TDS data support a (partially) diffusion limited retention behaviour for the LTS. For the HTS exposures, it clear that high thermal de-trapping rates are the determining factor for the D retention.

\begin{tabular}{|c|c|c|c|}
\hline Damage & Temperature scenario & Exposure time & Total D atoms retained \\
\hline $0 \mathrm{dpa}$ & HTS & $80 \mathrm{~s}$ & $0.7 \times 10^{16} \mathrm{D}$ \\
\hline $0 \mathrm{dpa}$ & LTS & $80 \mathrm{~s}$ & $0.5 \times 10^{16} \mathrm{D}$ \\
\hline $2 \mathrm{dpa}$ & HTS & $80 \mathrm{~s}$ & $1.9 \times 10^{16} \mathrm{D}$ \\
\hline $2 \mathrm{dpa}$ & LTS & $80 \mathrm{~s}$ & $2.1 \times 10^{16} \mathrm{D}$ \\
\hline $1 \mathrm{dpa}$ & LTS & $160 \mathrm{~s}$ & $3.6 \times 10^{16} \mathrm{D}$ \\
\hline $1 \mathrm{dpa}$ & LTS & $240 \mathrm{~s}$ & $4.9 \times 10^{16} \mathrm{D}$ \\
\hline
\end{tabular}

Table 2: Total $D$ retention from irradiated $W$ targets as determined by TDS.

\section{Conclusions}

The irradiation has resulted in significant enhancement of the hydrogenic retention of the tungsten. The exact retention behaviour is still very much determined by the $\mathrm{W}$ surface temperature during plasma exposure to the high flux plasma in the Pilot-PSI experiment. Both NRA and TDS indicate that for higher temperatures $\left(\mathrm{T}_{\mathrm{W}} \geq\right.$ $650 \mathrm{~K})$, the retention is determined by thermal de-trapping rates. For lower temperatures $\left(\mathrm{T}_{\mathrm{W}} \leq 480 \mathrm{~K}\right)$, it is diffusion that plays a more dominant role in determining the total retention although there are also indications of a plasma fluence dependence at these surface temperatures but further data is needed to investigate this fully.

The magnitude of the enhancement of retention due to the irradiation damage is also strongly dependent on the surface temperature during plasma exposure. The higher surface temperatures resulted in greater 
enhancement of the retention. The maximum enhancement was found at $950 \mathrm{~K}$, where retention was enhanced by a factor of 15-23. A surprising result given that significant retention in $\mathrm{W}$ has never previously been measured at this temperature. This could have serious implications for future fusion reactors that are expected to operate with the entire wall at these temperatures. Even if local concentrations saturate at $\sim 0.4$ at $\%$ as seen in this investigation, this still extrapolates to $\sim 6.3 \mathrm{~g}$ of tritium per square meter for a $1 \mathrm{~cm}$ thick $\mathrm{W}$ plasma facing component, assuming the wall is permeated, which will be the case in a steady-state reactor.

TDS spectra show us that the irradiation is creating two types of defects in the $\mathrm{W}$ that are influence the hydrogenic retention. The lower temperature desorption peaks $(500-600 \mathrm{~K})$ likely represent single vacancies with a trap energy of 1.0-1.4 eV. The high temperature desorption peak $(850 \mathrm{~K})$ is likely associated with the formation of micro-voids or vacancy clusters in the $\mathrm{W}$ with a trap energy of $\sim 2.1 \mathrm{eV}$. It is these high energy traps that contribute significantly to the retention at high surface temperatures during the plasma exposure.

Although this study is meant as a proxy for neutron damage, one key difference is the rate of damage produced in the material. For neutrons, the damage rate is very slow, many orders of magnitude slower than the damage production of the high energy $\mathrm{W}$ ions. This could be an important difference in terms of how the irradiation damage is distributed between vacancy formation and void/vacancy cluster formation. Also, the differences between simultaneous and sequential irradiation and plasma exposure could also play a strong role in determining how irradiation damage affects the retention properties of $\mathrm{W}$. There is still significant work to be done along these lines but this investigation has shown that this remains a concern and important topic even beyond the next generation devices all the way to the commercial fusion reactor operating with hot walls.

\section{Acknowledgements}

This work, supported by the European Communities under the contract of the Association EURATOM/FOM, was carried out within the framework of the European Fusion Programme with financial support from NWO. The views and opinions expressed herein do not necessarily reflect those of the European Commission.

The technical assistance of M. Fußeder and J. Dorner during the MeV irradiations is gratefully acknowledged. 


\section{References}

[1] B. Tyburska, V. Kh. Alimov, O. V. Ogorodnikova, K. Schmid, K. Ertl, J. Nucl. Mater. 395 (2009) 150

[2] W.R. Wampler, R.P. Doerner, Nuclear Fusion 49 (2009) 115023.

[3] G.M. Wright, D.G. Whyte, B. Lipschultz, J. Nucl. Mater. 390-391 (2009) 544.

[4] I. Takagi, S. Watanabe, S. Nagaoka, K. Higashi. Fusion Sci. Technol., 41(2002) 897.

[5] M. Fukumoto et al. J. Nucl. Mater. 390-391 (2009) 572.

[6] B.M. Oliver, T.J. Venhaus, R.A. Causey, F.A. Garner, S.A. Maloy. J. Nucl. Mater. 307-311 (2002) 1418.

[7] Q. Xu, T. Yoshiie, H. C. Huang, Nucl. Instrum. and Methods in Phys. Res. Sect. B 206 (2003) 123-126.

[8] G. J. van Rooij, V. P. Veremiyenko, W. J. Goedheer, et al. Appl. Phys. Lett. 90 (2007) 121501.

[9] A.E. Shumack, V.P. Veremiyenko, et al. Phys. Rev. E 78 (2008) 046405.

[10] M. Mayer, E.Gauthier, K. Sugiyama, and U. von Toussaint, Nucl. Instr. Meth. B 267 (2009) 506-512

[11] M. Mayer. Simnra user's guide. Technical report, Report IPP 9/113, Max Planck-Institut fur Plasmaphysik, 1997.

[12] R.A. Causey, C.L. Kunz and D.F. Cowgill, J. Nucl. Mater. 337-339 (2005) 600.

[13] G.N. Luo, W.M. Shu and M. Nishi, Fusion Engineering and Design 81 (2006) 957.

[14] J.P. Sharpe, R.D. Kolasinski, M. Shimada, P. Calderoni, R.A. Causey, J. Nucl. Mater. 390-391 (2009) 709.

[15] V. Kh. Alimov, J. Roth, R.A. Causey et al. J. Nucl. Mater. 375 (2008) 192.

[16] G.M. Wright et al. J. Nucl. Mater. 390-391 (2009) 610.

[17] G.M. Wright, E Alves, L C Alves, N P Barradas, P A Carvalho, R Mateus, J Rapp, Nuclear Fusion 50 (2010) 055004.

[18] R. Frauenfelder. J. Vac. Sci. Technol. 6 (1969) 388.

[19] A.A. Haasz, M. Poon, J.W. Davis, J. Nucl. Mater. 266 (1999) 520.

[20] W.M. Shu, Appl. Phys. Lett. 92 (2008) 211905.

[21] M. Poon, A.A. Haasz, J.W. Davis, J. Nucl. Mater. 374 (2008) 390.

[22] Eleveld and Van Veen, JNM 212-215 (1994) 1421

[23] Yu. M. Gasparyan, A.V. Golubeva, M. Mayer, A.A. Pisarev, and J. Roth, J. Nucl. Mater. 390-391 (2009) 606 


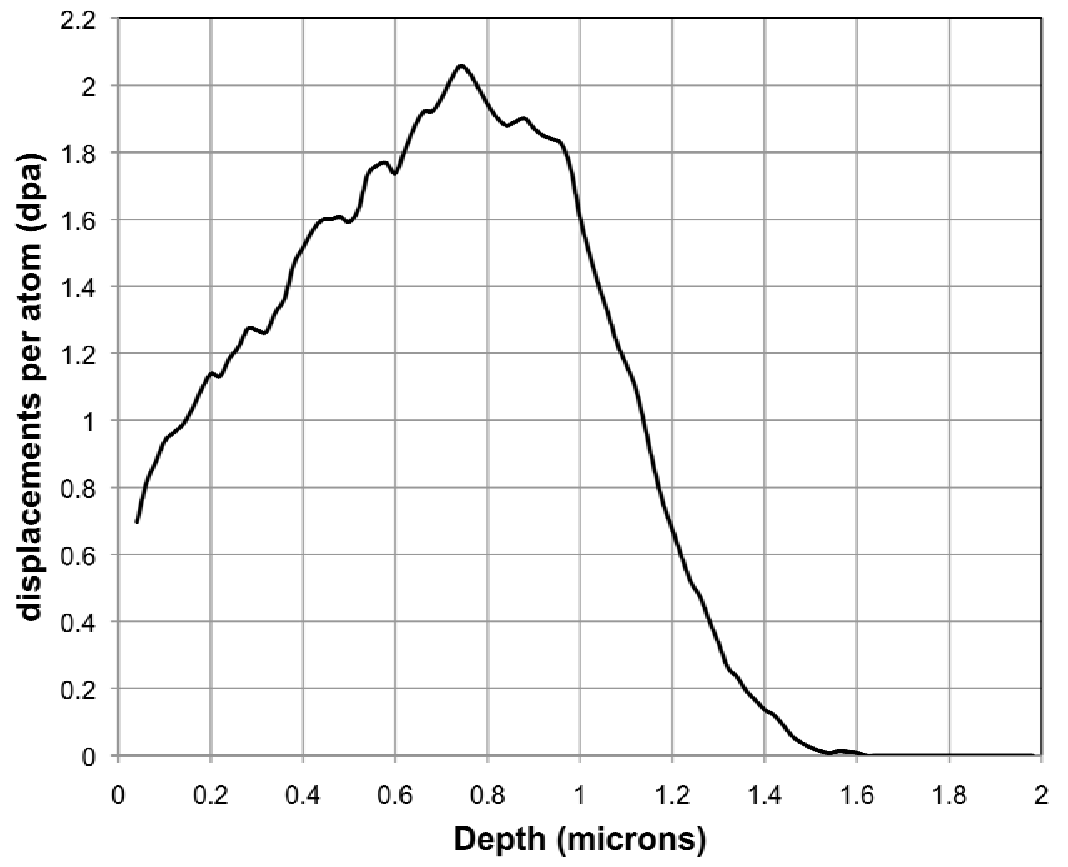

Figure 1: TRIM simulation of damage distribution (for 2 dpa peak case) with $E_{d}=40 \mathrm{eV}$. 


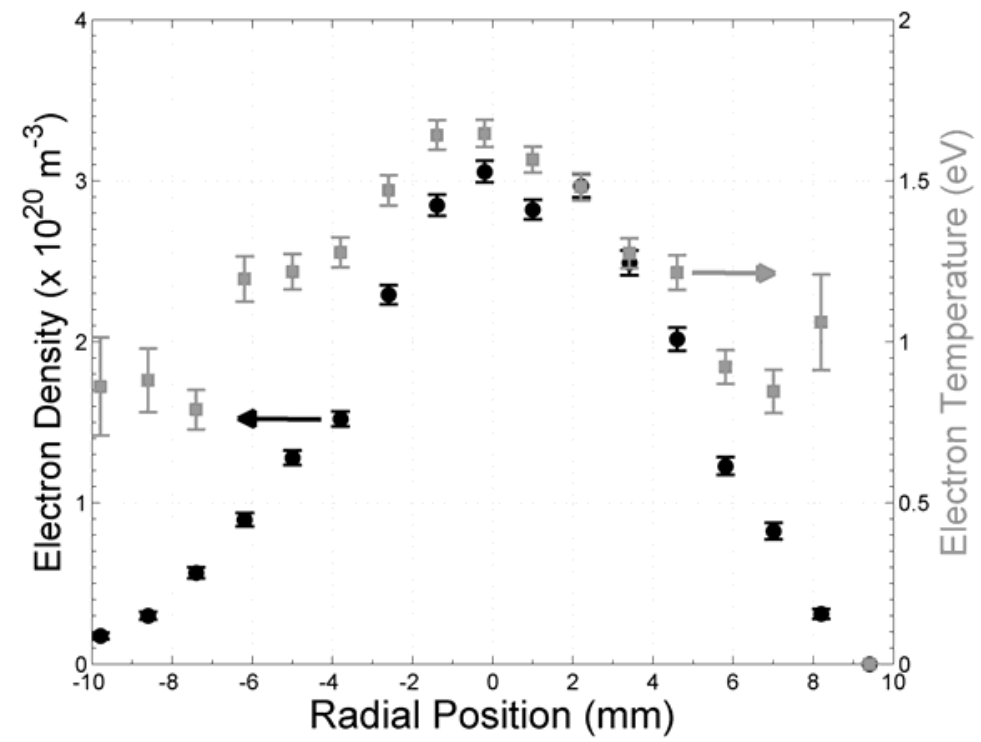

Figure 2: $n_{e}$ and $T_{e}$ radial distribution for plasma exposure in Pilot-PSI. 


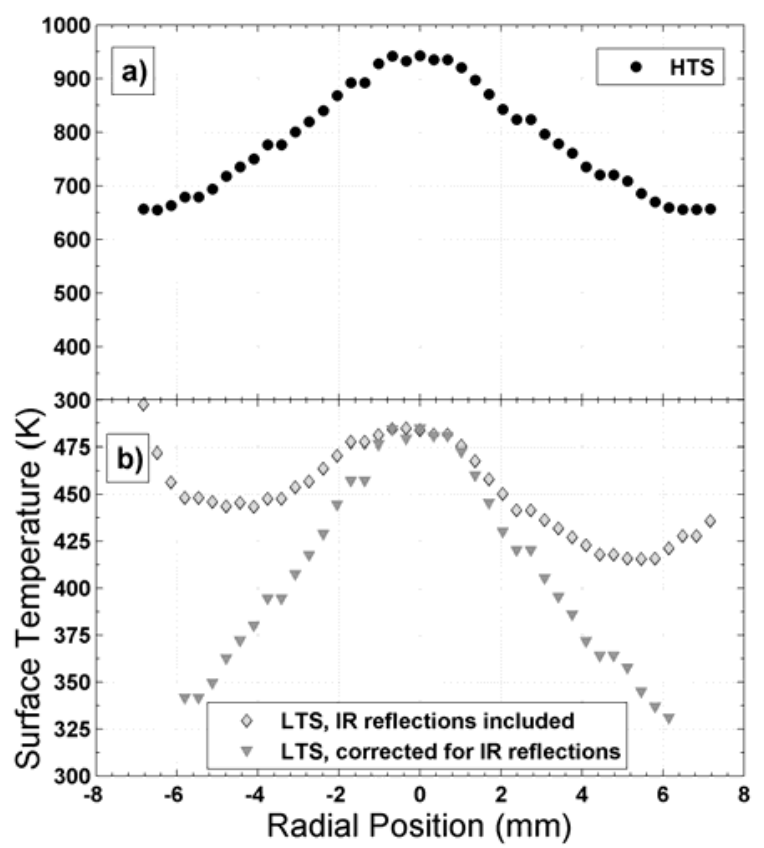

Figure 3: Temperature profile for a) high temperature and b) low temperature scenarios. 


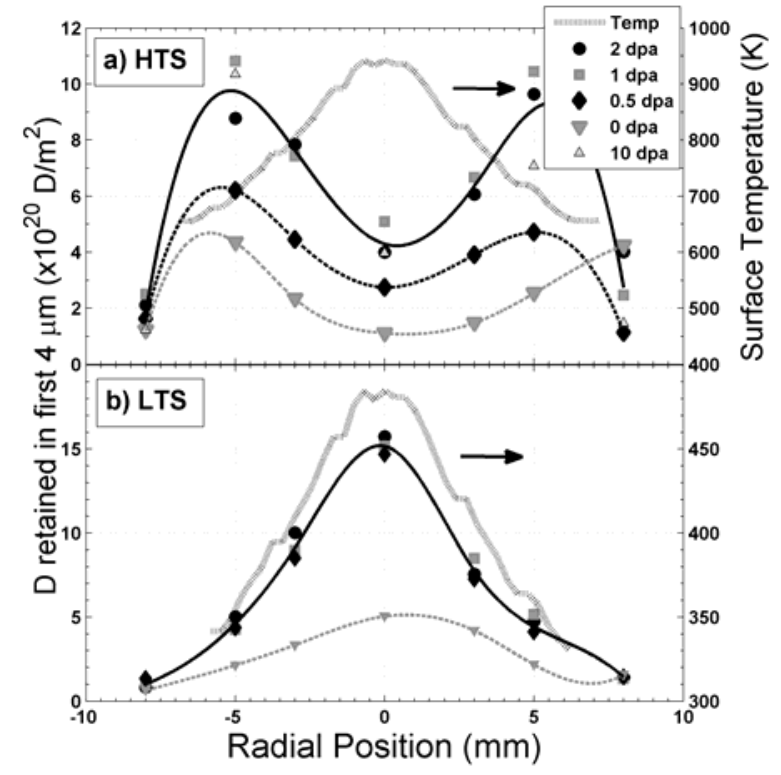

Figure 4: D retention in the first $4 \mu \mathrm{m}$ as a function of radial position for high temperature (top) and low temperature (bottom) scenarios. Lines are spline fits to guide the reader's eye. 


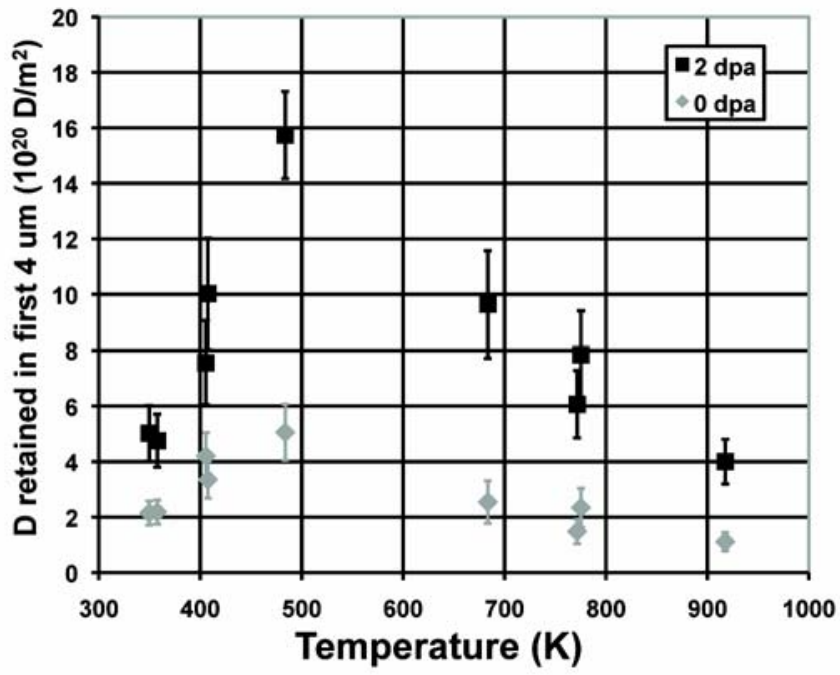

Figure 5: Local D retention in the first $4 \mu \mathrm{m}$ as a function of local tungsten surface temperature. Note that other parameters such as flux and fluence are not constant for this data (refer to table 1). 


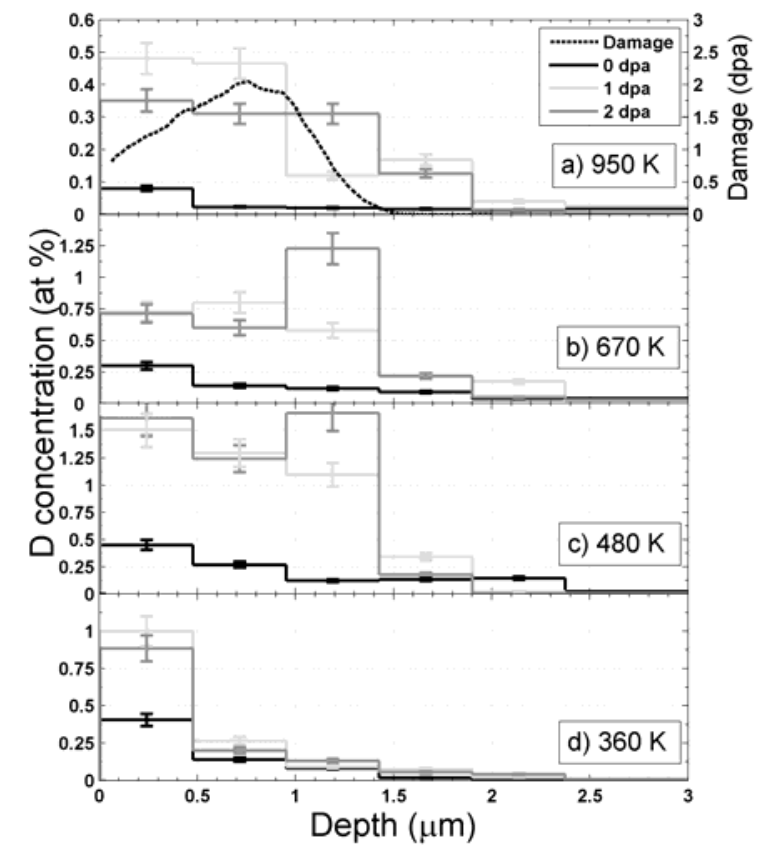

Figure 6: D depth profiles for irradiated and unirradiated targets at a) $r=0 \mathrm{~mm}$ (center) in the high temperature scenario, b) $r=5 \mathrm{~mm}$ in the high temperature scenario, c) $r=0 \mathrm{~mm}$ in the LTS, and d) $r=5 \mathrm{~mm}$ in the LTS. Damage scale is for the 2 dpa case is shown in a). 


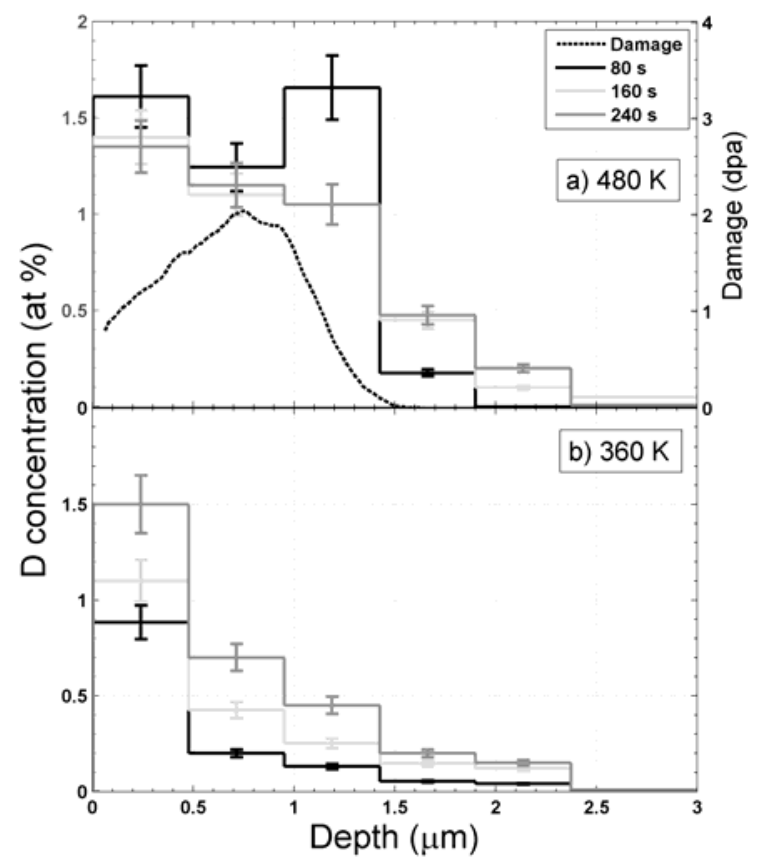

Figure 7: The D depth profiles for increasing exposure times/fluences for a) $r=0 \mathrm{~mm}$ (center) and b) $r=5 \mathrm{~mm}$ in the LTS. 


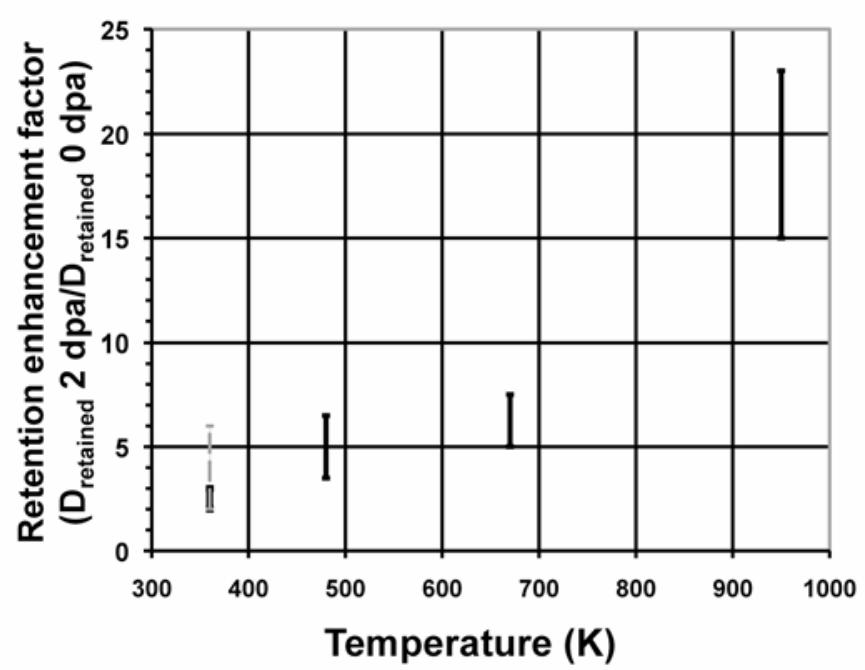

Figure 8: The retention enhancement factor as a function of tungsten temperature. The dashed grey line represents estimated enhancement factor if irradiated zone is fully permeated. 


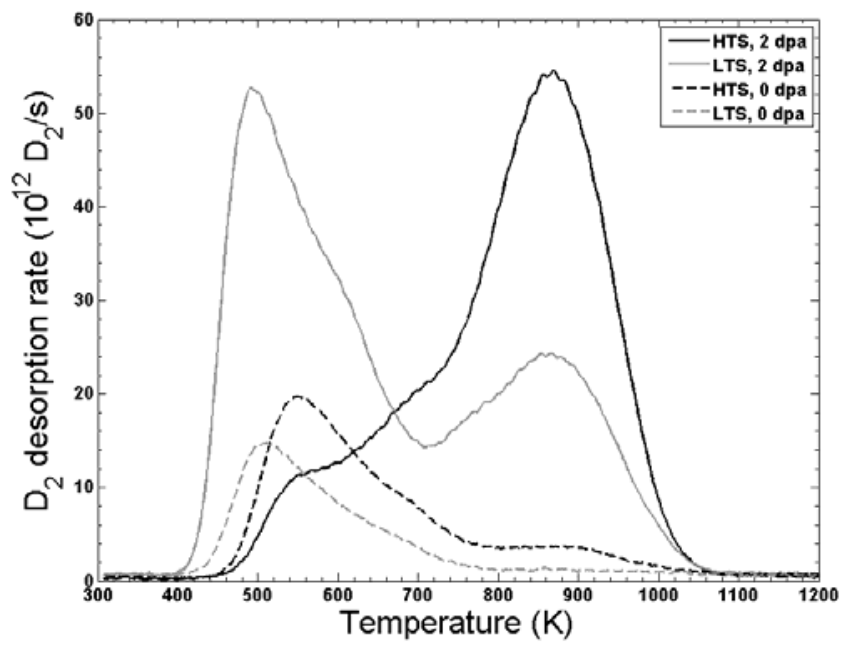

Figure 9: Thermal desorption spectra for irradiated ( 2 dpa) and unirradiated ( 0 dpa) W targets in the low temperature (LTS) and high temperature scenarios (HTS). 


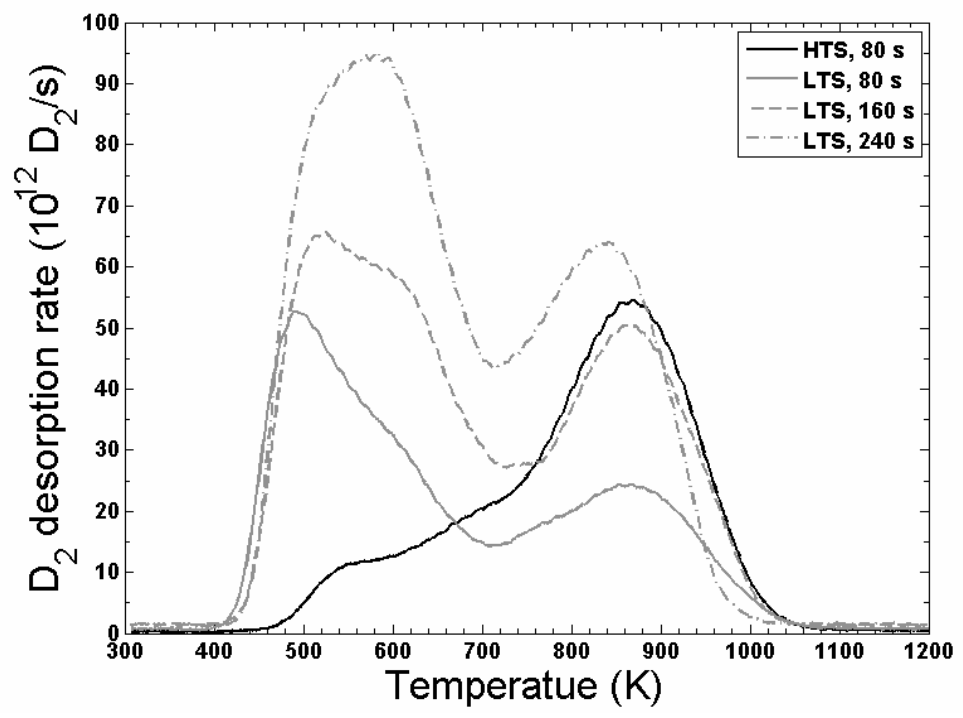

Figure 10: TDS spectra of irradiated W targets exposed for longer times in the LTS with a spectrum from an irradiated $W$ target in the HTS for comparison. 